\title{
OAs PARA O ENSINO DE CÁLCULO: POTENCIALIDADES DE TECNOLOGIAS 3D
}

Raiane Lemke - PPGECMT/UDESC - raiane.lemke@ gmail.com

Ivanete Zuchi Siple - PPGECMT/UDESC - ivazuchi@gmail.com

Elisandra Bar de Figueiredo - CCT/UDESC- elis.b.figueiredo@ gmail.com

Resumo Neste trabalho apresentamos as potencialidades das tecnologias da geometria dinâmica do software GeoGebra e da impressão 3D na criação de objetos de aprendizagem (OAs) para o ensino de funções de várias variáveis visando contribuir com a integração da tecnologia no Ensino de Cálculo com práticas inovadoras. Os OAs desenvolvidos virtualmente e fisicamente estão sendo utilizados na disciplina de Cálculo Diferencial e Integral II (CDI 2), nos cursos de Licenciatura em Matemática e Engenharias do Centro Tecnológico da Universidade do Estado de Santa Catarina. Os resultados dessa prática têm contribuído no processo de ensino, auxiliando na representação gráfica de superfícies, na interseção de superfícies e na visualização de curvas de nível.

Palavras-chave: Objetos de aprendizagem. Cálculo. GeoGebra 3D. Impressão 3D.

\section{LOS FOR TEACHING CALCULUS: THE POTENTIAL OF 3D TECHNOLOGIES}

Summary: In this paper we present the potential of the technologies of the software GeoGebra's dynamic geometry and of 3D printing to create learning objects (LOs) for the teaching of several variables functions aiming to contribute to the integration of technology in the teaching of Calculus with innovative practices. The virtually and physically developed LOs are being used in the discipline of Differential and Integral Calculus II (DIC 2) of the Mathematics Teaching and Engineering programs of the State University of Santa Catarina. The results of this practice have contributed in the teaching process, helping to graphically represent surfaces, surface intersections and contour curves.

Keywords: Learning objects. Calculus. GeoGebra 3D. 3D printing.

\section{Introdução}

Os objetos de aprendizagem (OAs) são procedentes de pesquisas desenvolvidas nos grupos de pesquisa PEMSA ${ }^{1}$ e NEPesTEEM $^{2}$ da Universidade do Estado de Santa Catarina-UDESC, sobre a utilização das tecnologias no ensino de matemática. Uma das pesquisas, em andamento, tem por objetivo a criação de um observatório e laboratório de práticas inovadoras no ensino de Cálculo, e um dos seus resultados foi o desenvolvimento de OAs que estão sendo utilizados na disciplina de Cálculo Diferencial e Integral II (CDI 2).

No ensino de Cálculo de funções de várias variáveis, a visualização desempenha um papel importante para a compreensão de diversos conteúdos, como integrais duplas, gráficos de funções de duas variáveis e diferenciais de volume. Neste contexto, entendemos visualização como sendo "o processo de formação de imagens (mentais, ou

\footnotetext{
${ }^{1}$ Grupo de Pesquisa em Educação Matemática e Sistemas Aplicados ao Ensino

${ }^{2}$ Núcleo de Estudo e Pesquisa em Tecnologia Educacional e Educação Matemática

V. $14 \mathrm{~N}^{\mathrm{o}} 1$, julho, 2016
} 
com lápis e papel, ou com o auxílio de tecnologias) usando essas imagens de forma eficaz para a descoberta e compreensão da matemática."(ZIMMERMANN; CUNNINGHAM, 1991 apud FLORES; WAGNER; BURATTO, 2012, p. 34). Ademais, "consideramos que a visualização associada às tecnologias digitais proporciona novos cenários para a exploração e investigação matemática, se tornando um importante elo entre o estudante e o objeto em estudo." (OLIVEIRA, 2014, p. 37).

Diante disso, o uso de OAs, no ensino de Cálculo são recursos interessantes nos processos de ensino e aprendizagem, haja vista que entendemos como OA, qualquer recurso como maquetes, imagens, fotos, vídeos, animações, simulações, arquivos de texto, páginas de internet, quando utilizados como recursos que apoiam tais processos (SANTOS, 2007).

Os recursos desenvolvidos pelo grupo contemplam aplicativos dinâmicos usando o ambiente do GeoGebra 3D e maquetes físicas impressas usando a tecnologia da impressão 3D. Aqui, exploraremos as potencialidades dessas ferramentas para o desenvolvimento dos recursos que apoiam a prática do professor de Cálculo.

\section{Potencialidades do Geogebra 3D e da Impressora 3D}

O software GeoGebra combina a facilidade de utilização de um software de geometria dinâmica com certas características de um Sistema de Álgebra Computacional - CAS, possibilitando trabalhar de maneira dinâmica com várias representações de um objeto matemático, no caso específico das representações de funções de duas variáveis. Além disso, é um software de código aberto sob a licença $\mathrm{GNU}^{3}$ e disponível para diversas plataformas, inclusive para dispositivos móveis.

Escuder e Furner (2011) apontam algumas razões para a escolha desse software: ele é uma fonte livre, aberta, multiplataforma, disponível em vários idiomas, dinâmico, tem uma extensa e muito ativa comunidade internacional de usuários que apoiam uns aos outros com materiais didáticos e suporte técnico na utilização do programa. Segundo Preiner (2008), embora o GeoGebra fosse inicialmente desenvolvido com o objetivo de deixar os alunos a explorarem e descobrirem conceitos matemáticos por conta própria, acabou por ser também uma ferramenta muito útil e conveniente para o professor criar seus próprios materiais didáticos.

O software, recentemente lançado na versão 3D, oferece diferentes representações, tais como algébrica, gráfica e numérica, interligadas de maneira dinâmica, possibilitando uma ampla gama de recursos para que os professores possam idealizar as suas próprias concepções de OAs no ensino de Cálculo. Para os OAs desenvolvidos exploramos as potencialidades das representações gráficas das funções de duas variáveis, a interseção de superfícies, curvas no espaço, projeções nos planos coordenados e simulação dinâmica dessas representações.

Para representar graficamente uma superfície no Geogebra 3D pode-se usar as expressões implícita, explícita ou paramétrica. Por exemplo, considere uma esfera centrada na origem de raio 2. Implicitamente, digita-se $x^{2}+y^{2}+z^{2}=4$ no campo de entrada. Explicitamente, entra-se, no campo de entrada, com as funções $\sqrt{4-x^{2}-y^{2}}$ e $-\sqrt{4-x^{2}-y^{2}}$ e, na forma paramétrica, por meio do comando disponível no GeoGebra

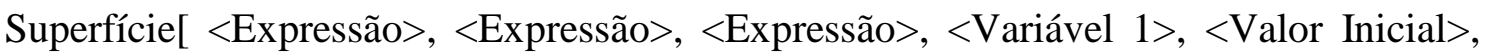
$\langle$ Valor Final $\rangle,\langle$ Variável 2>, <Valor Inicial $\rangle$, $\langle$ Valor Final $\rangle$ ]. No caso da esfera

${ }^{3}$ Licença Pública Geral, designação da licença para software livre.

V. $14 \mathrm{~N}^{\mathrm{o}} 1$, julho, 2016 
considerada, temos Superfície[2 $2 \cos (\mathrm{t}) \operatorname{sen}(\mathrm{u}), 2 \cos (\mathrm{t}) \cos (\mathrm{u}), 2 \operatorname{sen}(\mathrm{t}), \mathrm{t}, 0,2 \mathrm{pi}, \mathrm{u}, 0$, pi]. Essas três representações, respectivamente, estão ilustradas na Figura 1.

\begin{tabular}{|l|c|c|}
\hline Implícita & Explícita & Paramétrica \\
\hline
\end{tabular}

Figura 1 - Esferas

Também é possível representar gráfica e algebricamente as curvas de interseção de superfícies. Por exemplo, considere um cone circular reto ${ }^{4}$, centrado na origem, ao longo do eixo $z$. Para obter as curvas de interseção entre esse cone e os planos $x=1$ e $y=1$, digita-se, no campo de entrada, o cone na sua forma explícita $\left(\sqrt{x^{2}-y^{2}} \mathrm{e}\right.$ $-\sqrt{x^{2}-y^{2}}$ ) e os planos $x=1$ e $y=1$. Para obter as interseções, utiliza-se o comando Interseção[ 〈Objeto〉, 〈Objeto〉]. Assim, nesse caso supondo que a parte superior do cone seja nomeada como $a$ e o plano $x=1$ como $g$, digita-se Interseção[a, g], conforme ilustra a Figura 2. O usuário pode selecionar outros planos, obtendo assim diferentes curvas de interseção. O mesmo procedimento pode ser adotado com qualquer outra superfície explícita.

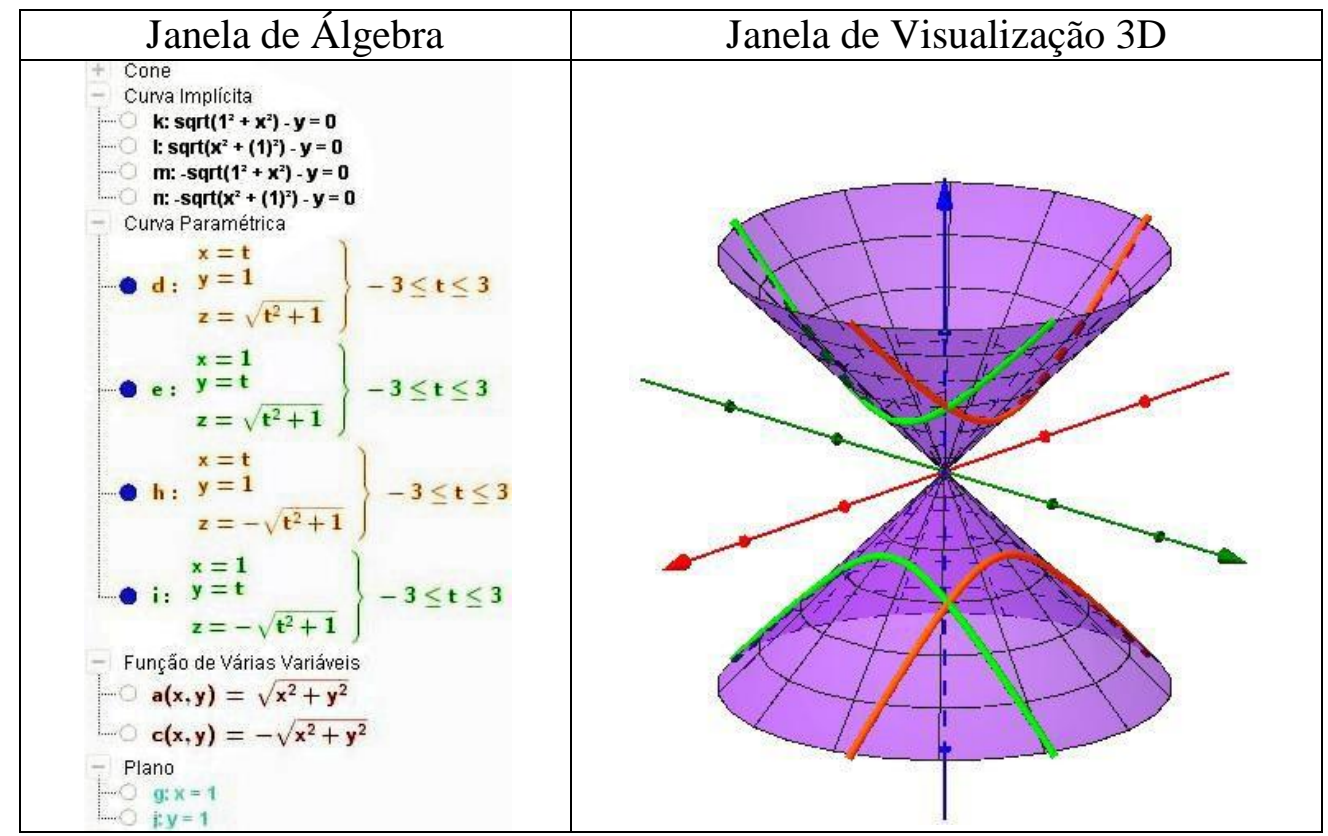

Figura 2 - Cone e interseções

A possibilidade de combinar o virtual com o real amplia o leque de exploração do OA, haja vista os diferentes enfoques de visualizações e manipulações oriundas desses ambientes. Como no grupo de pesquisa há a disponibilidade da impressora 3D

\footnotetext{
${ }^{4}$ Disponível em: http://ggbm.at/Ej2s5Y4B

V. $14 \mathrm{~N}^{\mathrm{o}}$ 1, julho, 2016
} 
Cliever $\mathrm{CL1}^{5}$ de fabricação nacional. Ela trabalha com arquivos em formato STL modelados em softwares de modelagem 3D, no nosso caso o Blender ${ }^{6}$. Exploramos as potencialidades dessa ferramenta em gerar maquetes físicas dos OAs desenvolvidos no GeoGebra 3D, tais como os cortes do cone, curvas de interseção de superfícies ${ }^{7}$, paraboloides $^{8}$, cone oblíquo ${ }^{9}$ e paraboloide hiperbólico ${ }^{10}$, como ilustrados nas Figuras 3, $4,5,6$ e 7 .

\begin{tabular}{|l|l|l|}
\hline Maquete 3D & Maquete 3D & Geogebra 3D \\
\hline & &
\end{tabular}

Figura 3 - Cone interceptado

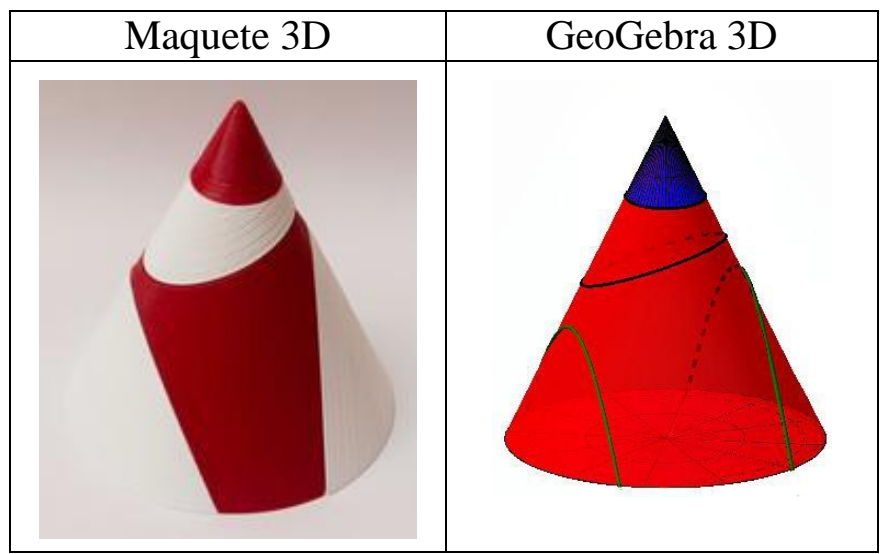

Figura 4 - Cone com interseções

\begin{tabular}{|c|c|}
\hline Maquete 3D & GeoGebra 3D \\
\hline & \\
\hline
\end{tabular}

Figura 5 - Paraboloide

\footnotetext{
${ }^{5}$ Cliever CL1: http://www.cliever.com.br/produto/impressora-3d-cliever-cl1-1

${ }^{6}$ Blender: <http://www.blender.org/>

${ }^{7}$ Disponível em: http://ggbm.at/Ej2s5Y4B

${ }^{8}$ Paraboloides em geral: http://ggbm.at/XCb9ghKv e sólidos formados por paraboloides e planos: http://ggbm.at/m9MasY8D

${ }^{9}$ Disponível em: http://ggbm.at/XutvPb4h

${ }^{10}$ Disponível em: http://ggbm.at/g5RUEAhD

V. $14 \mathrm{~N}^{\mathrm{o}} 1$, julho, 2016
} 


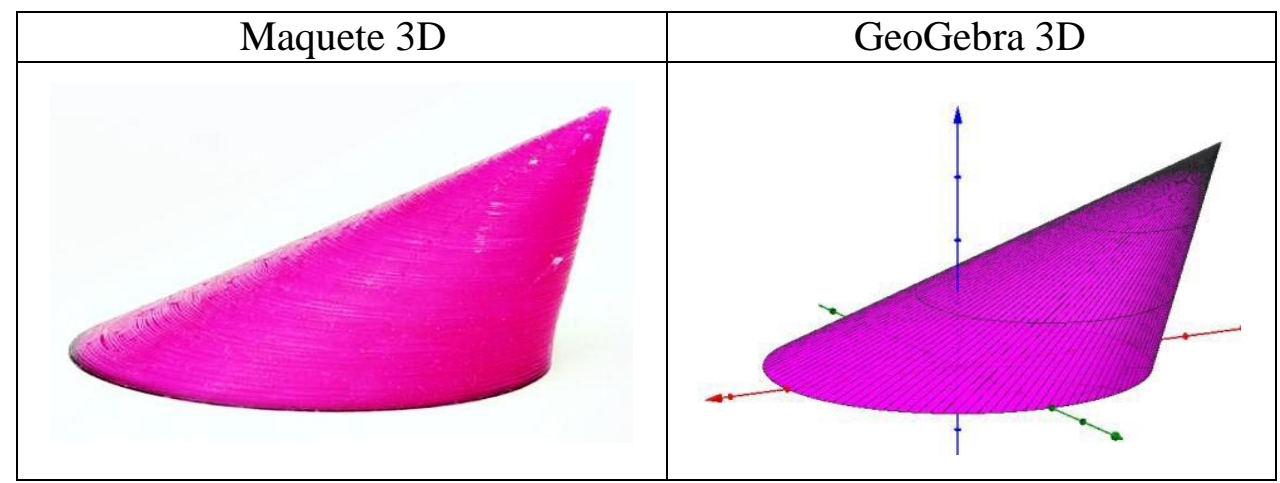

Figura 6 - Cone oblíquo

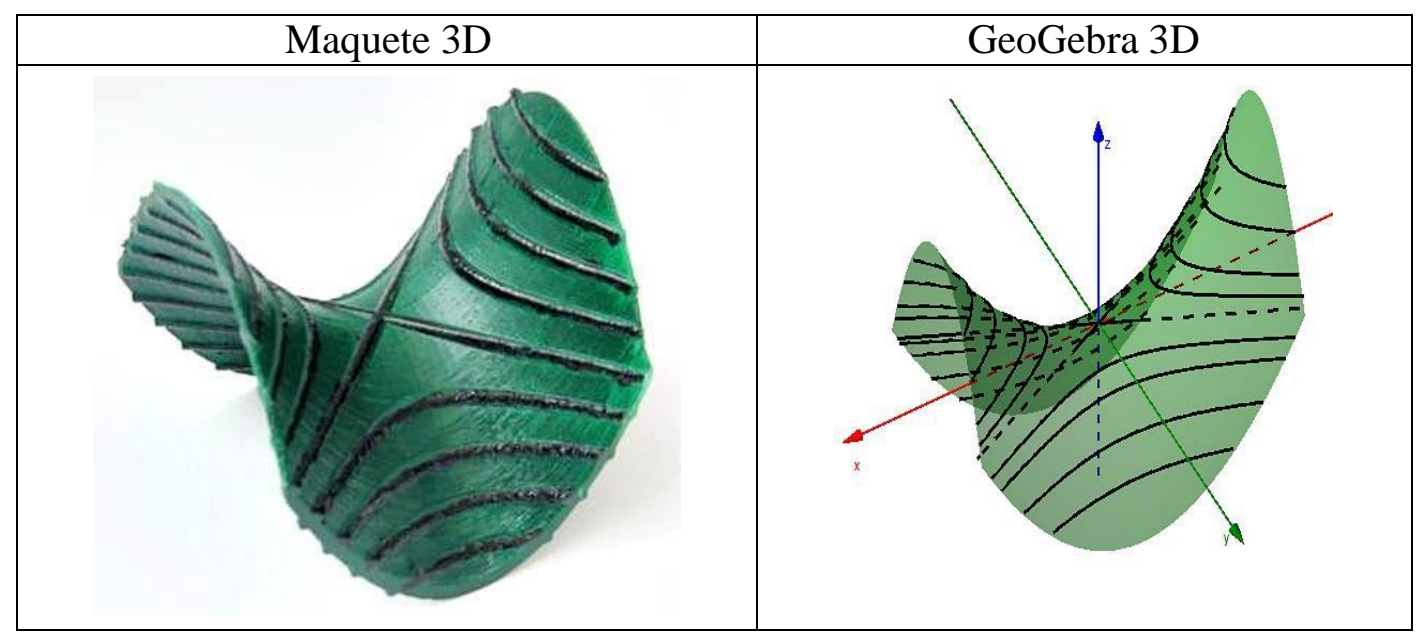

Figura 7 - Paraboloide hiperbólico e curvas de interseção

A impressão 3D foi uma tecnologia desenvolvida, na década de 80, como uma opção rápida para prototipagem de projetos e atualmente vem sendo usada por diversos setores, inclusive na educação. $\mathrm{Na}$ área acadêmica, tem-se tornado rapidamente uma opção acessível para produção de objetos físicos que podem ser utilizados como OAs em diversas áreas e em vários níveis de ensino. Nessa pesquisa exploram-se as potencialidades da impressora 3D para o ensino de Matemática.
A evolução acontece de forma constante e com ela vêm novidades que se adequarão à classe dos ferramentais educacionais. As formas de interação tomarão outros enfoques, tornando os conteúdos cada vez mais acessíveis, compreensíveis e ilustráveis. Algumas ferramentas que ampliam os conceitos de modelagem e tridimensionalidade palpáveis são os scanners e as impressoras 3D. No caso da impressora, reproduzindo, por meio de um software, um projeto estrutural desenvolvido pelo usuário em ambiente virtual, trazendo-o ao ambiente físico (TEODORO; LOPES, 2013, p. 98).

Segundo Slawkovsky (2012) a representação de objetos 3D não é privilégio dos tempos atuais, os educadores têm usado modelos tridimensionais elaborados com diferentes materiais por séculos, como se constata nos Elementos Euclides que já incluíam objetos dobrados feitos de papel para auxiliar na demonstração de objetos tridimensionais. Ainda a autora destaca que o objeto físico propicia o entendimento diferenciado do objeto virtual que dependendo da sua complexidade é quase impossível de ser abstraído.

Na sequência exploraremos o OA de diferencial de volume esférico, que não é um objeto trivial, modelado no GeoGebra e na impressora 3D. 


\section{Diferencial de volume esférico: Conexão entre GeoGebra e impressora 3D}

No cálculo de integrais triplas, dependendo das superfícies envolvidas, o uso do sistema de coordenadas esféricas pode facilitar a descrição do sólido, dos limitantes e a resolução da integral. Nesse sistema de coordenadas, o diferencial de volume é uma cunha esférica definida por

$$
E=\left\{\left.(\rho, \theta, \phi)\right|_{\rho_{1}} \leq \rho \leq \rho_{2}, \theta_{1} \leq \theta \leq \theta_{2}, \phi_{1} 1 \leq \phi \leq \phi_{1} 2\right\}
$$

cujo volume é $d V=\rho^{2} \sin \phi d \rho d \phi d \theta$.

Portanto, a equivalência entre a integral tripla no sistema de coordenadas cartesianas e esféricas é dada por:

$$
\int_{x_{0}}^{x_{1}} \int_{y_{0}}^{y_{1}} \int_{z_{0}}^{z_{1}} f(x, y, z) d z d y d x=\int_{\theta_{1}}^{\theta_{2}} \int_{\phi_{1}}^{\phi_{2}} \int_{\rho_{1}}^{\rho_{2}} f(\rho, \theta, \phi) \rho^{2} \sin \phi d \rho d \phi d \theta
$$

Na Figura 8 estão ilustrados o OA do diferencial de volume esférico que foi modelado dinamicamente no GeoGebra e sua maquete que foi impressa na impressora $3 \mathrm{D}$.

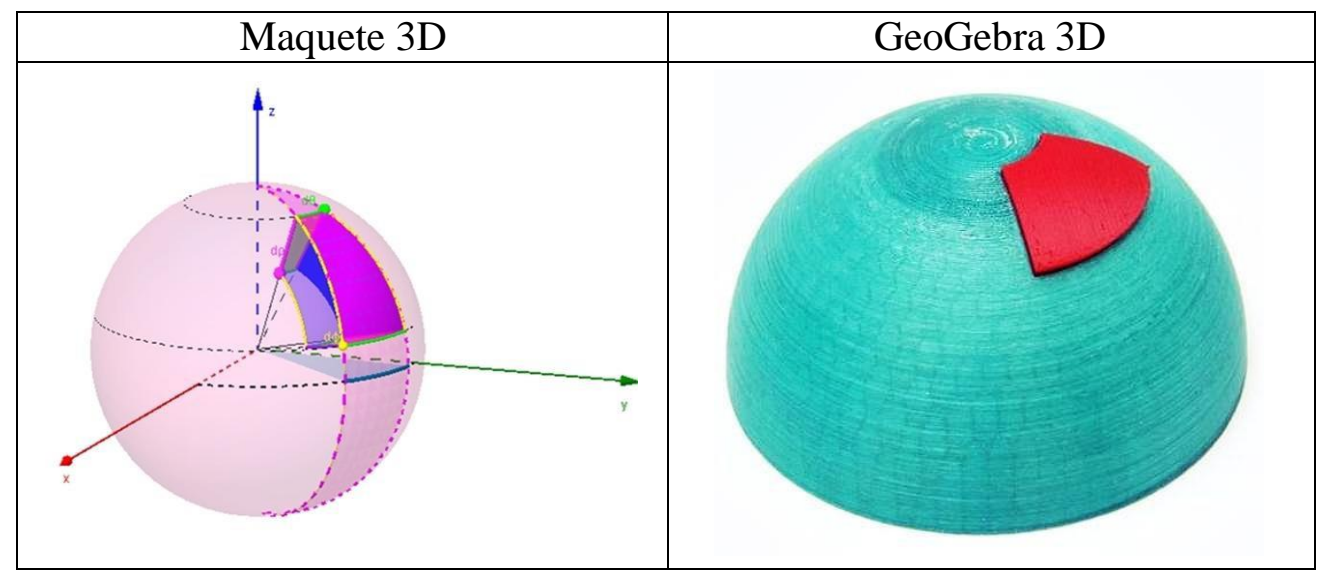

Figura 8 - Diferencial de volume esférico impresso e no GeoGebra

No ambiente do GeoGebra, desenvolvemos dois aplicativos ${ }^{11}$ abordando o diferencial de volume esférico, um estático e outro dinâmico. O estático tem por objetivo mostrar virtualmente o formato do diferencial do volume da esfera, considerando-se a dificuldade de visualização desse "pedacinho da esfera". Para isso, utilizamos os recursos do GeoGebra de girar, ampliar ou reduzir de maneira a ter o melhor ângulo de visualização, conforme ilustra a Figura 9, explorando os meridianos e paralelos.

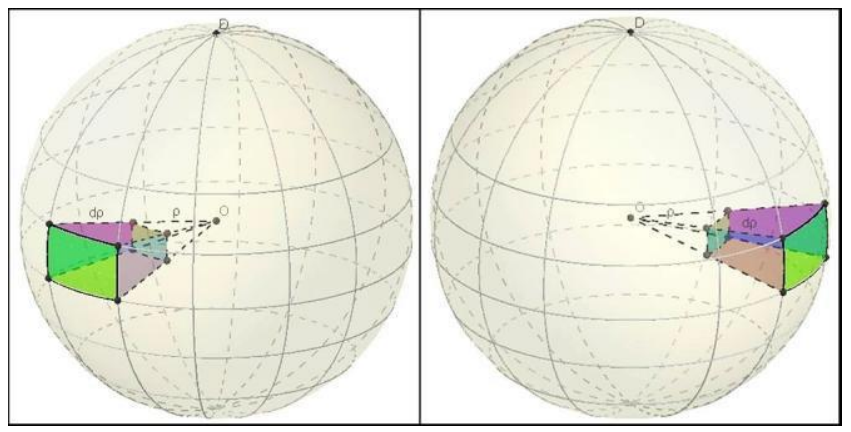

Figura 9 - Diferencial de volume esférico estático

${ }^{11}$ Disponíveis em: http://ggbtu.be/m1461487 
Outra opção interessante do GeoGebra é usar a visualização com o recurso da projeção para óculos 3D. Essa opção encontra-se na Janela de Visualização 3D, conforme ilustrado na Figura 10.

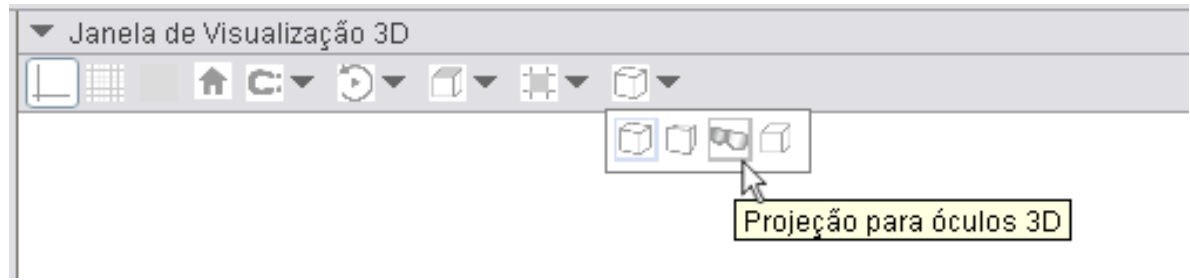

Figura 10 - Ferramenta para projeção do óculos 3D

Se houver a disponibilidade de óculos anáglifos, popularmente conhecidos como óculos azul e vermelho (Figura 11), esse OA é muito atrativo visualmente com esse recurso, pois facilita a visualização tridimensional do objeto .
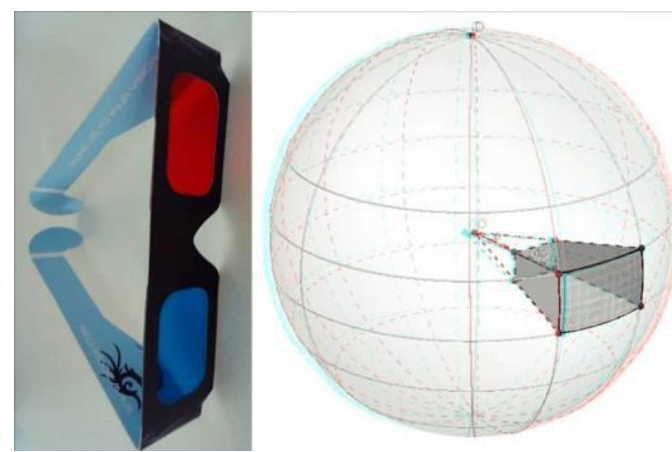

Figura 11 - Potencialidade óculos 3D no GeoGebra

No OA dinâmico o usuário pode fazer simulações da variação dos ângulos $\phi$ e $\theta$ e do raio $\rho$. Para isso, arrastam-se os pontos verde, amarelo e roxo, respectivamente. Conforme o usuário faz o movimento dinâmico desses pontos ele poderá observar o que acontece com o diferencial do volume esférico, conforme ilustra a Figura 12.

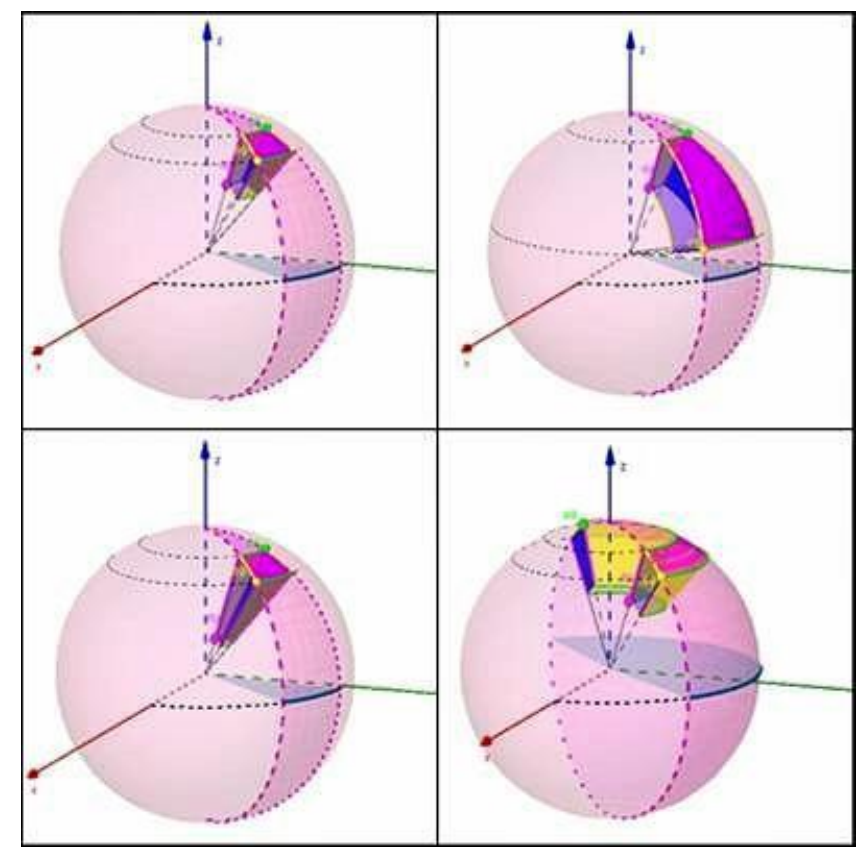

Figura 12 - Diferencial de volume esférico dinâmico 
Esse aplicativo pode ser utilizado pelo professor para introduzir integrais em coordenadas esféricas explorando a variação dos ângulos, bem como o vetor no espaço o raio da esfera. Também pode ser utilizado para mostrar as curvas de níveis da esfera. Outra sugestão, ainda, é explorar a passagem do diferencial de volume de coordenadas cartesianas para coordenadas esféricas, explorando as projeções na esfera. Esse OA também pode ser explorado pelo aluno, tendo em conta que ele é disponibilizado gratuitamente no GeoGebraTube, podendo ser explorado online ou offline, inclusive em plataformas móveis. Assim, o aluno poderá explorar tal recurso tanto no ambiente de sala de aula quanto extraclasse.

Outra sugestão para o ensino do diferencial de volume esférico é a combinação entre a maquete virtual e a maquete física (vide Figura 13). A maquete física auxilia na percepção tátil do objeto, haja vista que mesmo com os recursos tridimensionais das ferramentas ainda é uma tela bidimensional. $\mathrm{O}$ aluno pode explorar os cortes, as curvas de interseção, tanto na esfera quanto no diferencial. Por outro lado, o diferencial de volume da maquete física é estático (não podemos alterar as dimensões), nesse sentido a combinação dos dois objetos pode contemplar diferentes percepções dos alunos.

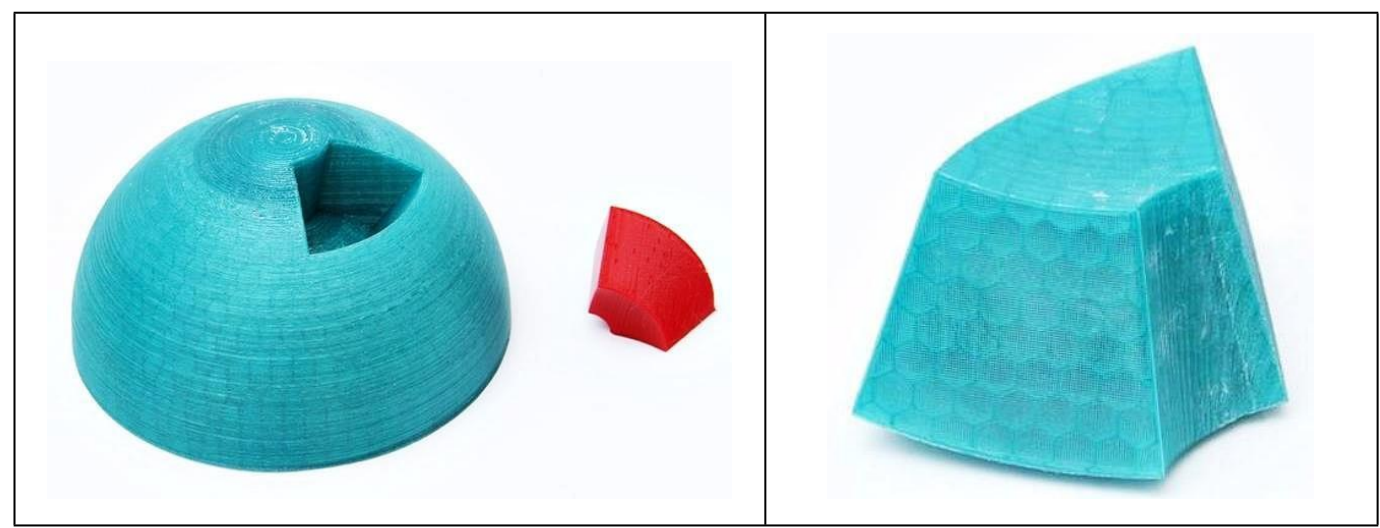

Figura 13 - Maquete física do diferencial de volume esférico de CDI 2.

Na sequência apresentamos relatos do uso desses OAs na prática de professores

\section{Diferencial de volume esférico: em sala de aula}

Desde o primeiro semestre de 2015 alguns professores, incluindo as autoras, que ministram a disciplina de CDI 2, do Centro de Ciências Tecnológicas - CCT-UDESC, estão utilizando esses objetos em suas práticas docentes para introduzir o conteúdo de integrais triplas no sistema de coordenadas esféricas. Para tal, alguns professores usam o OA virtual, outros o físico, ou ambos. Nas discussões do grupo uma das dificuldades, relatadas pelos professores em suas práticas, era trabalhar com a questão do diferencial do volume em coordenadas esféricas, dispendendo um tempo considerável para fazer a representação gráfica desse objeto, no ambiente lápis e papel, para depois, em sala de aula, transpor para o quadro negro e ainda assim não obtendo uma boa representação que facilitava a compreensão dos alunos. Sabemos que representar um objeto tridimensional num quadro, que é bidimensional, não é fácil, além do mais é sempre uma figura estática. Com a disponibilização desse OA dinâmico o quadro negro passou a ter movimento. 
como recurso o quadro e giz, fazendo um esboço geométrico do diferencial de volume, utilizava eixos cartesianos de forma física e canetas para auxiliar na minha explicação, e os alunos tinham que "imaginar" a variação do $\theta, \phi$ e $\rho$. Além disso, como nem sempre minha representação geométrica ficava boa no quadro, eu entregava aos alunos uma folha com um desenho do diferencial de volume para facilitar a visualização deles.

(...) No meu ponto de vista, o uso do aplicativo enriqueceu minha aula, pois facilitou minha explicação por ser algo dinâmico. Bastava mover os pontos para os alunos verificarem a variação (acréscimos/decréscimos) nas variáveis $\theta, \phi$ e $\rho$ e assim construir o diferencial de volume. Ao mover os pontos era possível observar que o volume do diferencial de volume também estava variando.

Acredito que os alunos ficaram satisfeitos com a explicação, pois alternei o tradicional do quadro e giz com um recurso tecnológico (que é sempre atrativo, é algo novo para eles) e, além disso, não fizeram questionamentos em relação à fórmula para o cálculo do volume então deduzida. As dúvidas, no momento da resolução de exercícios eram concentradas à transformação de um sistema de coordenadas para outro, enxergar a curva intersecção (LEMKE, 2015, p.77).

Na visão de alguns professores a utilização apenas do OA virtual ainda deixava lacunas na visualização do diferencial de volume esférico. Uma maneira de potencializar o OA foi concebê-lo também fisicamente. Para isso, foi impresso o artefato físico e utilizado em práticas. No contexto do ensino de CDI 2 possibilitou o confronto da representação no quadro de giz e na maquete física (Figura 14), pois propiciou aos alunos manipularem a maquete, observando os cortes, as variações dos ângulos e do raio do diferencial da esfera.

\section{Considerações}

A combinação da disponibilidade de objetos de aprendizagem para o Cálculo com uma metodologia adequada pode propiciar práticas inovadoras, instigando o aluno a realizar conjecturas, fazer simulações e confrontar as hipóteses numa situação problema. Temos a convicção que apenas a inserção do recurso tecnológico não proporciona uma nova prática, sendo necessária uma reflexão de como esse AO pode contribuir para uma melhor aprendizagem dos alunos.

Os OAs desenvolvidos no GeoGebra 3D e na impressora 3D possibilitam uma simulação de uma situação de investigação difícil quando restrita ao ambiente lápis e papel, fato que pode evitar a aprendizagem baseada na reprodução automática de fórmulas, muitas vezes desprovidas de significado conceitual. Além disso, Os OAs podem apoiar as diferentes modalidades de Educação: presencial, on-line ou mista. Os OAs virtuais podem ser disponibilizados tanto em computadores em rede como off-line, podendo ser acessados também por meio dos dispositivos móveis.

Frisamos que os OAs desenvolvidos estão em constante processo de evolução. Tal evolução é oriunda tanto dos feedbacks das experimentações realizadas em sala de aula quanto da própria evolução das ferramentas. Portanto, podemos dizer que nossos OAs não são recursos prontos e acabados, são protótipos e a sua evolução será produto tanto do avanço da tecnologia quanto da sua aplicação nos processos de ensino e aprendizagem, envolvendo a contribuição de vários autores - alunos, professores e pesquisadores. 


\section{Agradecimentos}

Os autores agradecem a FAPESC - Fundação de Amparo à Pesquisa e Inovação do Estado de Santa Catarina pelo apoio financeiro ao Grupo de Pesquisa PEMSA .

\section{Referências}

ESCUDER, A.; FURNER, J. M. The Impact of GeoGebra in Math Teachers' Professional Development. Electronic Proceedings of the Twenty-third Annual International Conference on Technology in Collegiate Mathematics, Denver, Denver, Colorado, March 17-20, 2011. Disponível em: < http://archives.math.utk.edu/ICTCM/VOL23/S113/paper.pdf > Acesso em: 29 abr. 2016.

FLORES, Cláudia Regina; WAGNER, Débora Regina. BURATTO, Ivone C. Freitas; Pesquisa em visualização na educação matemática: conceitos, tendências e perspectivas. In: Educ. Matem. Pesq., São Paulo, v.14, n.1, pp.31-45, 2012. Disponível em: < http://revistas.pucsp.br/index.php/emp/article/view/8008/6827> Acesso em 29 abr. 2016.

LEMKE, Raiane. Objetos de aprendizagem para o ensino de funções de duas variáveis: um diferencial dinâmico. 2015. TCC (graduação) - Universidade do Estado de Santa Catarina, Curso de Licenciatura em Matemática, Joinville, 2015.

OLIVEIRA, Fabio Luiz de. A Produção de Conhecimento Matemático acerca de Funções de Duas Variáveis em um Coletivo de Seres-humanos-com-mídias. 2014. Dissertação (mestrado) - Universidade Federal de Ouro Preto, Mestrado Profissional em Educação Matemática, Ouro Preto, 2014.

PREINER, J. Introducing Dynamic Mathematics Software to Mathematics Teachers: the Case of GeoGebra. (Dissertation in Mathematics Education Faculty of Natural Sciences). University of Salzburg, Salzburg, 2008.

SANTOS, L. M. Produção de significados para objetos de aprendizagem: de autores e leitores para a educação matemática. Dissertação (Mestrado) - Universidade Federal do Paraná, Curitiba, 2007.

SLAVKOVSKY, E. A. Feasibility Study For Teaching Geometry and Other Topics Using Three-Dimensional Printers. Dissertação (Mestrado) - Universidade de Harvard, Cambridge, USA, 2012.

TEODORO, João Vitor; LOPES, José Marcos. Evolução e perspectivas da tecnologia em sala de aula e na formação docente. In: Revista Educação e Fronteiras On-Line, Dourados/MS, v.3, n.8, p.91- 104, mai./ago. 2013. Disponível em: $<$ http://www.periodicos.ufgd.edu.br/index.php/ educacao/article/viewFile/3209/pdf_170> Acesso em: 29 abr. 2016. 